\title{
BMJ Open Randomised controlled clinical trial of a structured cognitive rehabilitation in patients with attention deficit following mild traumatic brain injury: study protocol
}

\author{
Norhamizan Hamzah, ${ }^{1}$ Vairavan Narayanan, ${ }^{2}$ Norlisah Ramli, ${ }^{3}$ \\ Nor Atikah Mustapha, ${ }^{4}$ Nor Adibah Mohammad Tahir, ${ }^{4}$ Li Kuo Tan, 3 \\ Mahmoud Danaee, ${ }^{\circ}$ Nor Asiah Muhamad, ${ }^{6}$ Avril Drummond, ${ }^{7}$ \\ Roshan das Nair, ${ }^{\oplus, 9}$ Sing Yau Goh, ${ }^{10}$ Mazlina Mazlan ${ }^{1}$
}

To cite: Hamzah $\mathrm{N}$,

Narayanan V, Ramli N, et al. Randomised controlled clinical trial of a structured cognitive rehabilitation in patients with attention deficit following mild traumatic brain injury: study protocol. BMJ Open 2019;9:e028711. doi:10.1136/ bmjopen-2018-028711

- Prepublication history for this paper is available online. To view these files, please visit the journal online (http://dx.doi. org/10.1136/bmjopen-2018028711).

Received 28 December 2018 Revised 19 August 2019 Accepted 20 August 2019
Check for updates

(C) Author(s) (or their employer(s)) 2019. Re-use permitted under CC BY-NC. No commercial re-use. See rights and permissions. Published by BMJ.

For numbered affiliations see end of article.

Correspondence to Dr Mazlina Mazlan; mazlinamazlan@ummc.edu.my

\section{ABSTRACT}

Objectives To measure the clinical, structural and functional changes of an individualised structured cognitive rehabilitation in mild traumatic brain injury (mTBI) population.

Setting A single centre study, Malaysia.

Participants Adults aged between 18 and 60 years with mTBI as a result of road traffic accident, with no previous history of head trauma, minimum of 9 years education and abnormal cognition at 3 months will be included. The exclusion criteria include pre-existing chronic illness or neurological/psychiatric condition, long-term medication that affects cognitive/psychological status, clinical evidence of substance intoxication at the time of injury and major polytrauma. Based on multiple estimated calculations, the minimum intended sample size is 50 participants (Cohen's d effect size $=0.35$; alpha level of 0.05 ; $85 \%$ power to detect statistical significance; $40 \%$ attrition rate).

Interventions Intervention group will receive individualised structured cognitive rehabilitation. Control group will receive the best patient-centred care for attention disorders. Therapy frequency for both groups will be 1 hour per week for 12 weeks.

Outcome measures Primary: Neuropsychological Assessment Battery-Screening Module (S-NAB) scores.

Secondary: Diffusion Tensor Imaging (DTI) parameters and Goal Attainment Scaling score (GAS).

Results Results will include descriptive statistics of population demographics, CogniPlus cognitive program and metacognitive strategies. The effect of intervention will be the effect size of S-NAB scores and mean GAS T scores. DTI parameters will be compared between groups via repeated measure analysis. Correlation analysis of outcome measures will be calculated using Pearson's correlation coefficient.

Conclusion This is a complex clinical intervention with multiple outcome measures to provide a comprehensive evidence-based treatment model.

Ethics and dissemination The study protocol was approved by the Medical Research Ethics Committee
Strengths and limitations of this study

- To our knowledge, this is the first randomised controlled trial of a cognitive intervention in an adult mild traumatic brain injury (mTBI) population, conducted in a low/middle-income country (Southeast Asia region)

- A study from this region, with various ethnic groups, may better represent the study population and in turn add further knowledge on the pattern of the impairment following mTBI.

- This trial incorporates technology in the intervention arm consistent with the changing face of health service delivery in Malaysia, aiming at both resource efficiency and treatment effectiveness, although the tailored treatment approach is appropriate for the local setting.

- Owing to the paucity of scientific and clinical knowledge, this trial will also contribute to the evidence-based cognitive treatment model for the mTBI population.

- We anticipate challenges in the recruitment phase and with treatment compliance due to known and reported high attrition rate in the traumatic brain injury population.

UMMC (MREC ID NO: 2016928-4293). The findings of the trial will be disseminated through peer-reviewed journals and scientific conferences.

Trial registration number NCT03237676

\section{BACKGROUND}

Mild traumatic brain injury (mTBI) is defined as a traumatic injury that induces transient physiological disruption of the brain function. ${ }^{1}$ mTBI is often used interchangeably with concussion and is a clinical diagnosis. ${ }^{1}$ The most common aetiology in the low-income and middle-income countries is road traffic 
accident (RTA) that disproportionately affects young men (15-29 years of age)..$^{2-4}$ Statistically, 20-50 million people sustained non-fatal injuries worldwide as a result of RTA and with an increasing rate in the low/middle-income countries. $^{23}$

Cognitive deficit is rarely singular in mTBI. Commonly reported symptoms are attention, memory and executive function deficits, each with varying severity and recovery pattern. ${ }^{5-14}$ Specifically, attention deficit is extremely common in TBI. ${ }^{1516}$ Attention is known to be the basis of all other cognitive abilities. ${ }^{17}$ About $40 \%-60 \%$ of individuals with $\mathrm{mTBI}$ are reported to have attention deficits in the first 3 months postinjury. ${ }^{18}$ In the majority of individuals, resolution of mixed cognitive deficits begins in the first month and up to 1-year postinjury. ${ }^{5} 7111219-21 \mathrm{~A}$ proportion of this population often progresses to have chronic cognitive disability that is overlooked due to the initial 'mild' presentation. ${ }^{6}{ }^{22-25}$ At least one-third of survivors fail to return to full functional status at 6 months and may, indeed continue to have neurocognitive functional deficits beyond 1 year of injury. 512 25-29

\section{Cognitive rehabilitation in $\mathrm{mTBI}$}

Currently, there is no standard cognitive rehabilitation treatment for mTBI population. ${ }^{19}$ The heterogeneity of cognitive deficits, varied intervention methodology, different reporting style and variable treatment outcomes, 6172728 30-57 challenge professionals in standardising mTBI treatment. ${ }^{19}$ The early neuropsychological model of attention has already made the assumption that attention should be the focus of rehabilitation, even before more advanced cognitive skills are treated. ${ }^{33}$ In the last 20 years, various cognitive treatment approaches have been reported in the systematic reviews. ${ }^{34-37}$ These include remediation strategies, ${ }^{38-49}$ compensatory strategies ${ }^{50-57}$ and patient education intervention. ${ }^{6395358}$ These approaches are usually applied in combination, in order to optimise both cognitive and functional recovery. ${ }^{172728303133-38}$ In particular, treatment for attention deficits in TBI has been recommended at postacute (3 months) stage of trauma. ${ }^{28} 303444$ Methods of treatment included multidimensional approach, and tasks with hierarchical difficulty and complexity. ${ }^{30} 34$ Several studies also reported improved psychological outcome and coping of symptoms on those who received patient education and reassurance following mTBI. ${ }^{63536}$ However, these conclusions were based on a limited number of high-quality clinical trials. The consensus was for more robust clinical trials with larger sample sizes, with well-described complex intervention and standardised reporting methods. ${ }^{1934-374446}$

Delivery of cognitive rehabilitation emphasises six principles: (1) intervention that is theory driven and meaningful, (2) intervention is task specific with increasing complexity relevant to individual needs, (3) the need to regularly practice skills acquired, (4) progress monitoring to tailor to individual's needs, (5) generalisation of learnt strategies to apply in real-life skills and (6) realworld adaptation to ensure success. ${ }^{17} 4959$ A practical, widely accepted treatment approach with the application of evidence-based treatment principles, may represent a comprehensive treatment model in treating patients with mTBI with cognitive deficits. A large randomised trial is required to support this hypothesis.

\section{Clinical, imaging and functional outcome measures in mTBI}

A combination of clinical, imaging and functional outcome measures is a comprehensive approach to analyse cognitive intervention that can make an impact in the clinical practice. Scientific reviews and guidelines have recommended the use of neuropsychological assessment as an appropriate clinical outcome measure. ${ }^{172728} 303133343637$ In adult mTBI, a test that is sensitive across various cognitive domains, ${ }^{21} 244143535760$ specific to population study, ${ }^{24} 4043$ has good validity and reliability, ${ }^{41515761-64}$ is cost effective and practical to use in a clinical setting ${ }^{5362-64}$ would be ideal.

The structural injury in mTBI, however, is too minuscule for detection through routine CT and MRI. ${ }^{65-67}$ Over the last 10 years, Diffusion Tensor Imaging (DTI) has become accepted as a non-invasive tool that is able to quantify microstructural brain changes in mTBI. ${ }^{2465-70}$ Changes in its parameters are indicative of microstructural remodelling at acute and chronic stages of injury, potentially explaining the persistence of symptoms that would otherwise be attributed to other causes. ${ }^{24} 65-70 \mathrm{~A}$ longitudinal DTI study may increase our understanding of the brain structural transformation in mTBI.

The most important outcome following mTBI is the ability for patients to return to their previous functional state and quality of life. Commonly used scales to measure disability and function are usually sensitive to cognitive deficits but not necessarily specific to the TBI population. ${ }^{39-415253}$ Many studies have also reported specific outcome measures for TBI that has good validity, reliability and practical in a clinical setting, ${ }^{71-79}$ such as Goal Attainment Scaling (GAS), ${ }^{71} 72-79$ Extended Glasgow Outcome Scale ${ }^{73}$ and Functional Assessment Measure. ${ }^{74}$

This trial evaluates a complex clinical intervention, which will provide evidence on the effect of cognitive rehabilitation in mTBI. The outcome measures include anatomical, clinical and functional aspects to provide a comprehensive evidence-based treatment model.

\section{METHODS}

\section{Study hypothesis}

We hypothesise that structured cognitive rehabilitation for attention deficits following mTBI will improve patients' cognitive function of attention compared with standard care.

\section{Study objectives}

The objectives are:

- To measure the clinical effect of a 12-week individualised structured cognitive rehabilitation, which addresses attention deficit and overall cognitive status.

- To analyse the effect of treatment on brain structures and functional changes. 


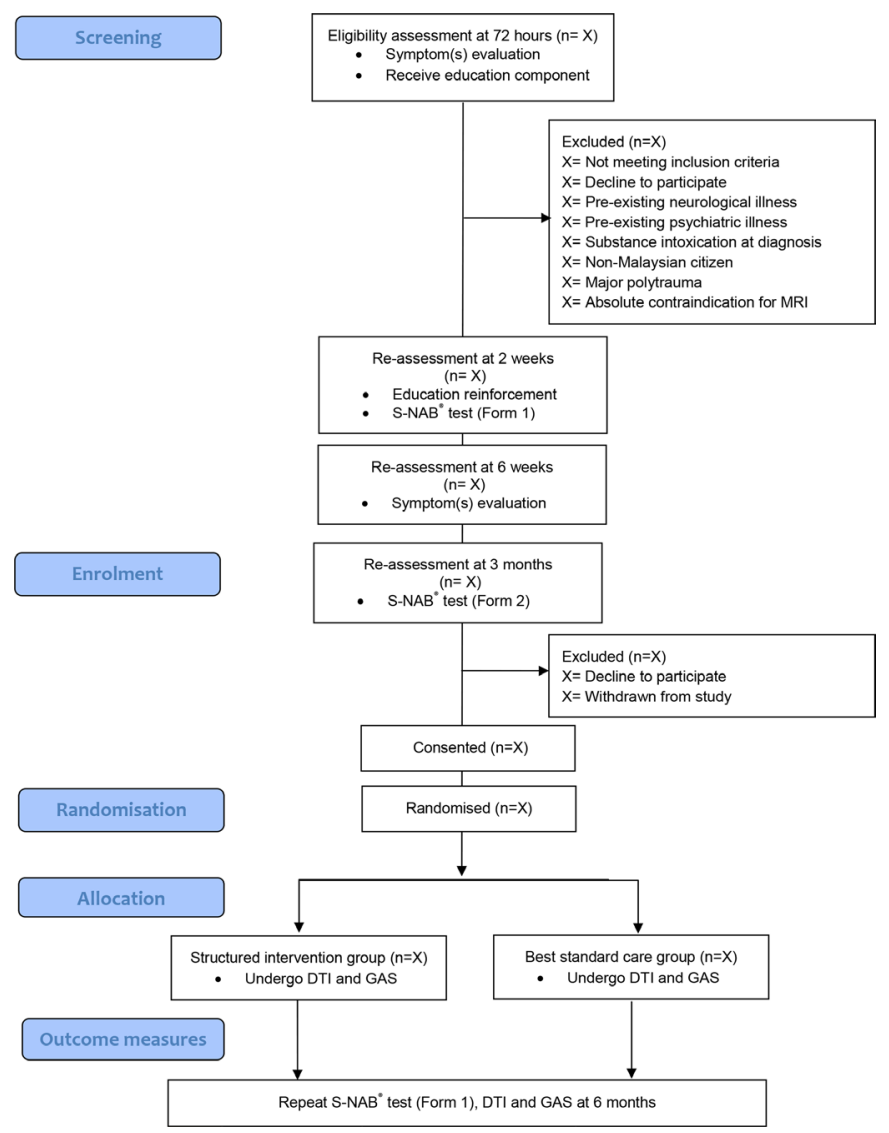

Figure 1 Flow chart showing the stages of recruitment in this study. DTI, Diffusion Tensor Imaging; GAS, Goal Attainment Scaling; S-NAB, Neuropsychological Assessment Battery-Screening Module.

- To correlate clinical effects following cognitive rehabilitation with structural brain changes and participants' overall functional outcomes.

\section{Design}

This will be a prospective double blind, randomised controlled trial with two parallel groups. The study design is summarised in figure 1.

\section{Participants and recruitment process}

This trial will be conducted at a single centre, University Malaya Medical Centre (UMMC), Malaysia. UMMC is a government-funded academic medical institution situated in the urban area of the nation's capital city Kuala Lumpur with a population of 1.76 million. Apart from providing acute medical services, this hospital is also a tertiary referral and training centre in Malaysia. UMMC also houses Department of Rehabilitation Medicine that provides the facility for this study. This department includes the main rehabilitation services (neurorehabilitation, spinal cord rehabilitation, prosthetic rehabilitation and orthotic rehabilitation, paediatric rehabilitation and cardiac rehabilitation) for both inpatient and outpatient setting. Other services also include return to work/ driving rehabilitation.
We will recruit participants through the emergency medicine department (ED), UMMC from 1 August 2017. ED physicians, radiologists and neurosurgeons will refer mTBI cases to a research assistant for recruitment. Potential cases will also be screened through the UMMC digital medical record system. Screening stages will be performed at 72 hours, 2 and 6 weeks following mTBI.

\section{Inclusion criteria}

mTBI is defined as physiological disruption of brain function as a result of trauma with symptoms of loss of consciousness $30 \mathrm{~min}$ or less, focal neurological deficit that may/may not be transient, altered mental state with Glasgow Coma Scale of 13-15 and loss of memory with post-traumatic amnesia not greater than 24 hours. The inclusion criteria for this study are mTBI as a result of RTA; adult aged between 18 and 60 years old; Malaysian resident; no previous history of head trauma; minimum of 9 years education; persistently abnormal Neuropsychological Assessment Battery-Screening Module (S-NAB) Attention Domain score at 3 months of mTBI; ability to give consent and willingness to comply with cognitive rehabilitation programme. Persistently abnormal S-NAB Attention Domain score is defined as Standard Score $<85$ (below average category) at screening phase and at enrolment phase as set by the NAB test manual (table 1).

\section{Exclusion criteria}

The exclusion criteria include pre-existing chronic illness that causes neurological symptoms or complications; severe comorbid neurological or psychiatric disorder; on long-term medication that alters or affects cognitive and psychological status; clinical evidence of substance intoxication at the time of injury; major polytrauma and absolute contraindications for MRI (metal or implant not compatible for MRI, claustrophobia) (table 1).

\section{Intervention}

Potential participants will undergo screening before enrolment and randomisation (figure 1). The education component will include reassurance on recovery, self-monitoring of symptom(s) and advice on gradual return to daily activities and physical exertion. Symptom(s) evaluation will include clinical review of physical, cognitive and psychological status. The first medical responder, that is, ED physicians will perform this review at 72 hours of injury. At 2 and 6 weeks after injury, a rehabilitation medicine physician who is not involved with the study (RP-1) will repeat the education component and symptom evaluation. Early treatment or referral to other medical specialty will be made if indicated during these reviews.

At 3 months after injury, potential participants will undergo a repeat of clinical review and S-NAB test. Eligibility criteria will include (1) an abnormal S-NAB Attention Domain score at 3 months post-mTBI or (2) deficits in more than one S-NAB domain, not including the attention domain. The concomitant domain deficit(s) will also 


\begin{tabular}{|c|c|c|c|}
\hline Criteria & IG & SG & HG \\
\hline \multicolumn{4}{|l|}{ Inclusion criteria } \\
\hline 18-60 years old of age & $\checkmark$ & $\checkmark$ & $\checkmark$ \\
\hline No previous history of head trauma & $\checkmark$ & $\checkmark$ & $\checkmark$ \\
\hline Minimum of 9 years education & $\checkmark$ & $\checkmark$ & $\checkmark$ \\
\hline Consented & $\checkmark$ & $\checkmark$ & $\checkmark$ \\
\hline mTBI as a result of motor vehicle accidents only & $\checkmark$ & $\checkmark$ & \\
\hline Abnormal S-NAB Attention Domain score at 3 months of mTBI & $\checkmark$ & $\checkmark$ & \\
\hline \multicolumn{4}{|l|}{ Willingness to comply with rehabilitation programme } \\
\hline \multicolumn{4}{|l|}{ Exclusion criteria } \\
\hline Pre-existing chronic illness or neurological or psychiatric condition & $\checkmark$ & $\checkmark$ & $\checkmark$ \\
\hline $\begin{array}{l}\text { On long-term medication that can alter or affect cognitive and/or psychological } \\
\text { status }\end{array}$ & $\checkmark$ & $\checkmark$ & $\checkmark$ \\
\hline Clinical evidence of alcohol intoxication at the time of injury & $\checkmark$ & $\checkmark$ & \\
\hline Major polytrauma (multiple bone fractures, nerve injury) & $\checkmark$ & $\checkmark$ & \\
\hline Absolute contraindication for MRI & $\checkmark$ & $\checkmark$ & \\
\hline
\end{tabular}

IG-individualised structured cognitive rehabilitation group.

HG, healthy control group; mTBI, mild traumatic brain injury; SG, standard care group; S-NAB, Neuropsychological Assessment BatteryScreening Module.

be evaluated on completion of therapy. The cognitive intervention will be conducted at the Neurorehabilitation Therapy Unit, Department of Rehabilitation Medicine, UMMC as an outpatient setting. Participants will be assigned to different treatment groups via the randomisation process. Written records of the intervention will be recorded and kept by the therapist of each treatment arm until treatment completion. This will include the participant's goals, symptom(s), cognitive strategy/method and participant's feedback.

\section{Individualised structured cognitive rehabilitation group}

Intervention group participants will receive a 2-part 12-week individualised structured cognitive rehabilitation. The first part will be Direct Attention Training, a deficit-oriented computer-based attention-training programme called CogniPlus. ${ }^{45}$ Each session last 30 min, once a week.

CogniPlus is a computer-based software programe with interactive multimedia approach for multiple attention cognitive training modules. The training programs are ALERT (focused and sustained attention), FOCUS (focused attention), VIG (sustained attention), SELECT (selective attention) and DIVID (divided attention). Each attention-training category is designed based on real-life scenarios. The screen graphics are three dimensional. This program has an artificial intelligence capacity that can automatically adapt to an individual's performance and alter the training difficulty level (hierarchical difficulty).

The second part of this intervention will be strategy approach (metacognitive awareness and compensatory strategy) performed after CogniPlus training. Metacognitive awareness includes feedback on the participant's CogniPlus performance to improve their awareness of impairment severity. This process is intended to regulate learning experience and in turn instil the practise of self-monitoring and self-regulation through learning activities. Compensatory strategy component involves instilling cognitive awareness in recognising impairment that is present in daily activities. This will be followed by the application of cognitive methods to ameliorate the deficit to maximise daily functioning. A participant will identify the deficit(s) and will apply problem-solving $\operatorname{method}(\mathrm{s})$ learnt from the therapist. Feedback and review of performance will be repeated in the next following therapy session. The metacognitive strategies applied will also be recorded in writing during the participant's feedback sessions. This session will last for $30 \mathrm{~min}$ and a will be conducted by a trained and certified occupational therapist (OT-1) in cognitive therapy and CogniPlus.

\section{Standard care group}

This group will receive the best standard care for attention disorders. This is a patient-centred cognitive therapy. It is based on a patient's complaint(s), symptom(s) and therapy aim(s) (self-realisation of deficits or guided by therapist). Symptom(s) management may include physical (eg, imbalance, fatigue and sleep dysregulation), psychological (eg, mild anxiety or depression) and cognitive (eg, forgetfulness). Referral to relevant service(s) may be required such as physiotherapy, return to work/driving rehabilitation and counselling. Compensatory strategy includes task-specific training (patient prioritised), for 
example, return to driving may involve driving simulation training, and visuospatial training. The frequency of sessions will be 1 hour per week, for 12 weeks. A trained OT in cognitive therapy (OT-2), who is not involved with the intervention group treatment, will conduct all the sessions (table 2).

\section{Control group}

This will consist of healthy individuals demographically matched for age, gender and education years to the intervention groups (table 1). They will undergo S-NAB, DTI imaging and psychological screening tools, which will include the Generalised Anxiety Disorder 7-item (GAD-7) and Patient Health Questionnaire-9 (PHQ-9). Their lifestyle aspects will also be reviewed and recorded (spiritual practice, diet, physical exercise, occupation and driving). The data will be collected for comparison purpose.

\section{Randomisation, consent and blinding}

Participants with mTBI who fulfil the study criteria will be randomised via computer-generated random permuted block assignment, gender stratified into equally proportioned intervention and control group numbers. The study schedule, procedures and blinding of coinvestigators are presented in table 2.

\section{Modification, withdrawal and unblinding within the intervention}

Participants can withdraw their consent from this study at any time and for any reason. Investigators can also withdraw a participant from the study if he/she becomes non-compliant with the treatment protocol. This includes poor treatment attendance (non-attendance of $>50 \%$ of total therapy sessions) or the participant's request for withdrawal from the study. We will also provide necessary treatment to participants who require immediate medical attention that is otherwise not part of the study intervention throughout the study duration. In the case where unblinding of a participant is necessary (eg, medical emergency), an investigator (MM) will be informed of the cause and stage of intervention. He/she may continue in the study and follow all study procedures. The participant will only be withdrawn from this study if the immediate treatment violates the study criteria. We will retain all of participant's data (although the participant is no longer blinded) up to the point of participant's removal from the study.

\section{Adherence strategies}

Adherence to treatment is encouraged for both groups. This will be achieved by providing: (1) participants with clear information on purpose, method and treatment goals during treatment sessions, (2) an appointment card with specific date and time of therapy sessions and (3) a reminder through phone calls a day before each therapy appointment and a week before DTI scan date.

\section{Outcome measures}

All measures will be performed at baseline and at 12 weeks of intervention after randomisation. The primary outcome measure for this study is the change in attention deficit and other cognitive domains within intervention groups and direct comparison of each intervention group with the healthy control group. This will be measured by NAB (PAR, Florida, USA). ${ }^{61}$ It consists of six modules: Screening Module and five Domain Specific Modules: Attention, Language, Memory, Spatial and Executive Function. This study will only apply the Screening Module (S-NAB) because it measures the same five functional domains similar or identical to the main NAB modules. It consists of 12 individual tests screening all five mentioned cognitive domains for adults aged 18-97 years, validated and sensitive for use in healthy and cognitively impaired brain injured population. ${ }^{24}{ }^{61-64} \mathrm{~S}-\mathrm{NAB}$ also provides two parallel assessment sets (Record Form 1 and Form 2) that will be applied in an alternate fashion to participants in both groups to avoid practice effect.

S-NAB Domain Attention test items and score are interpreted as a marker of an individual's attentional capacity, working memory, psychomotor speed, selective attention, divided attention and information processing. ${ }^{61}$ S-NAB has also been applied in our previous cohort study ${ }^{24}$ with good validation outcome in our Malaysian mTBI population.

The secondary outcome measures are microstructural white matter tract (WMT) parameters and GAS scores. The DTI MRI scan is a Siemens Magnetom Prisma 3T MRI (Siemens AG, Muenchen, Germany). This study will analyse fractional anisotropy (FA), mean diffusivity (MD) and radial diffusivity (RD) parameter changes at preintervention and postintervention. ${ }^{2465-70}$ These parameters quantify the direction and degree of tissue water diffusion within the WMT ${ }^{6566} \mathrm{FA}$, which measures the direction of the diffusion, is an index expressed in a range from 0 to 1 , with a higher score indicating a higher integrity of white matter consisting of highly parallel fibres. ${ }^{6566} \mathrm{MD}$ measures the average magnitude of the diffusion while RD quantifies pathology in the myelin. ${ }^{6566}$ Changes in the index values of the parameters at different injury timeline will indicate the pathological changes of the WMT.

The tool to measure functional goal outcome will be the GAS. ${ }^{77-79}$ The difficulty and importance of rehabilitation goals will be individually set according to his/her current levels of functional performance to reinforce realistic expectations. The sensitivity of GAS is increased by the quantifiable set goals relevant and specific to the participant. Each goal is rated on a 5-point scale and score is given on the extent to which a patient's individual goals are achieved in the course of the intervention. The overall GAS scores calculation will generate a standardised measure ( $T$ score) (mean of $50 \mathrm{SD} \pm 10$ ). The details of each goal outcome will be recorded in the GAS Record Sheet ${ }^{77-79}$ by a cognitive therapist from each study arm (OT-1 and OT-2) trained in GAS application. 


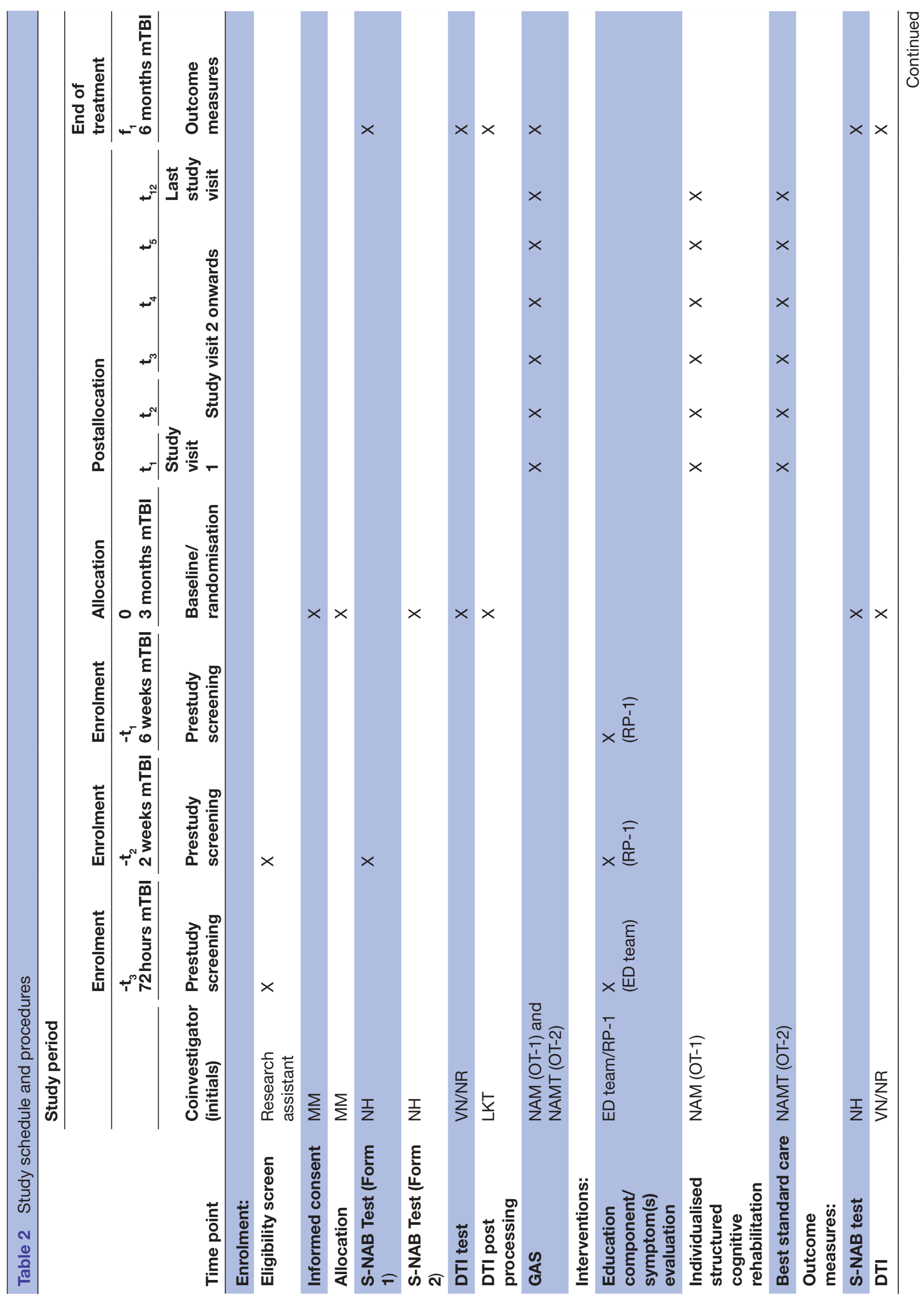

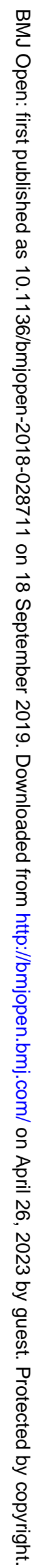


Another important factor to note is the participant's psychological status following mTBI. This study will also perform a screening of anxiety and depression symptoms by using GAD-7 and PHQ-9 screening tools at each study timeline. Participant's lifestyle changes/modifications such as spiritual practice, diet change, physical exercise, return to work/education, return to driving, litigation issues and insurance claims will also be reviewed and recorded. Although these parameters will not be part of the study outcome measure, they, however, remain relevant in influencing treatment adherence and outcome.

\section{Sample size and power calculation}

In order to fulfil our study objectives, we will base the intended sample size calculation on a previous study that had applied a similar treatment approach and which had a similar outcome measure to our study. ${ }^{40}$ This study applied the non-commercial statistical power analysis program $G^{*}$ Power V.3.1.9.2. An effect size of 0.58, which was the functional cognitive outcome of attention, ${ }^{40}$ is used to calculate the statistical power a priori. We will apply analysis of variance (ANOVA): repeated measures, within-between interaction, setting an alpha level of 0.05 , and approximately 10 participants will provide $89 \%$ power to detect a statistical significance. Recruitment is doubled $(n=20)$ for both arms and inflated to 28 to counter $40 \%$ attrition rate.

To have a bigger sample size, we, therefore, also decided on a more conservative effect size value and calculated the sample size through estimation of Cohen's d effect size value of 0.35 . By using similar statistical power analysis program, medium effect size Cohen's d of 0.35, setting an alpha level of 0.05 , approximately 38 participants will provide $85 \%$ power to detect statistical significance. Recruitment will be inflated to 50 participants to enable a $40 \%$ attrition rate.

Based on the multiple estimated calculations, the minimum intended sample size is 50 participants. From UMMC local data, a 12-month data collection is sufficient to yield the target sample size.

\section{Ethics considerations}

We will obtain written consent from participants. During consenting, participant will be provided with a patient information sheet detailing the purpose of study, reason for participation, study investigation and intervention methods, withdrawal from the study and contact details of investigators. Once consent is given, the consent form and all other documents with the participant's personal details will be stored immediately in a locked filing cabinet by the consent taker. This will be accessible only to a small number of investigators. Study ID codes will be allocated after consent is obtained and subsequent study documentation will only use the ID code.

Other issues included will be (1) early information sharing of treatment/investigation results in the event of incidental clinical findings that requires urgent treatment by other medical specialty, (2) treatment compliance, 
(3) cost of investigation and treatment, and (4) participant involvement in litigation issues. In the event of information sharing being required for medical reasons, the participant will be informed immediately, followed by referral to the relevant professional, either based at UMMC, or a different centre of choice. However, costs of further investigation or treatment that is not part of this study will not be funded from the study grants. Treatment compliance will be achieved through our adherence strategy. We strictly adhere to the privacy and confidentiality of participant's medical information. Any information sharing with a third party for various reasons will be managed in accordance with UMMC professional and legal code of conduct.

\section{Patient and public involvement}

We applied the Medical Research Council's Developing and Evaluating Complex Intervention: New Guidance (2006) and Multiphase Optimization Strategy (MOST) framework to guide the development and design of this study. The treatment approach was based on the relevant theoretical evidence whereas treatment approach was evinced through our systematic review, clinical experience and practice setting of interest. We conducted (1) a pilot study and (2) Expert Panel review to evaluate the study design and treatment method that may require further focus.

Participants in the pilot study were recruited in the testing of the treatment method, clinical practicality, fidelity of treatment and treatment compliance. We have additionally identified several components required for the optimisation of the intervention. These findings were also assessed by the Expert Panel reviewers.

The panel comprised of clinicians who were credentialed in brain injury management and cognitive rehabilitation, with minimum of 10 years clinical experience working in Malaysia. Panels were made up of seven rehabilitation medicine consultants, one neurosurgeon consultant, one neuroimaging consultant, five cognitive OT and one clinical psychologist. The focus of discussion was on the feasibility of structured cognitive rehabilitation for patients with mTBI in Malaysia, guided by the current evidence, current practise of cognitive rehabilitation in local setting, reviewers' clinical experience and our pilot study findings. A summary of the pilot study outcomes and Expert Panel recommendations are best illustrated in table 3.

Table 3 A summary of recommendations from pilot study findings and expert panel review

\section{Pilot study}

Design: a case-controlled study

Study components:

Non-randomisation - to identify participant's

willingness to attend therapy as a measure of good compliance.

Treatment application - treatment was given at early stage of injury (2 weeks postinjury) to measure the treatment effect vs spontaneous' recovery.

Treatment accessibility - outpatient hospital-based treatment is feasible.

Treatment compliance-high attrition rate (50\%), which compromised the treatment fidelity. Reasons for poor treatment compliance were:

- Treatment frequency and intensity (>1 hour/weekly for the first 3 months followed by monthly session the following 3 months)

- Mental fatigue.

- 'Unreadiness' to receive treatment.

- Treatment and transportation costs.

- Work demand (limited time off work and income lost).

Treatment method-clinical application of treatment was acceptable to participants.

Treatment effect-the application of effect

size measurement is consistent with MOST

recommendation.

Outcome measure application-S-NAB was able to measure score differences in its five domains. DTI parameters reported changes consistent with current literature evidence in $\mathrm{mTBI}$ population.

\section{Expert panel review}

Design: Randomisation was recommended in clinical trial design

Review components:

Fidelity of treatment

1. Clear information on purpose, method and treatment goals during treatment sessions.

2. An appointment card with specific date and time of therapy sessions.

3. A reminder through phone calls a week and a day before each therapy

4. Review at 72 hours, 2 weeks, 6 weeks and 3 months (baseline) to increase sensitivity towards participant selection, early medical intervention if required and to improve adherence.

Treatment method

1. As outpatient setting, with frequency 1 hour/week for 12 weeks duration.

2. Individualised treatment approach with standardisation through direct attention training and metacognitive strategy

3. To clarify the metacognitive strategies applied in therapy such as 'self-monitoring', self-instructional procedure', 'self-evaluation', 'rehearsal', 'self-pacing', 'positive self-statement', use of internal/ external strategy.

Outcome measure

Neuropsychological assessment as a practice standard

Guided individualised goals (GAS application) to standardise the functional goal outcome measurement for both groups.

DTI, Diffusion Tensor Imaging; GAS, Goal Attainment Scaling; MOST, Multiphase Optimisation Strategy; mTBI, mild traumatic brain injury; S-NAB, Neuropsychological Assessment Battery-Screening Module. 
Following the commencement of this study, the input from participants (experience, feedback and outcomes) will be recorded. The data and study materials will belong to UMMC, Malaysia. We will inform our participants of the result of the study following its completion, even if he/she did not complete the study unless he/she has requested no contact.

\section{Statistical analysis}

Descriptive statistics will be conducted on the data obtained from all groups to provide a demographic overview of our study population. A $\mathrm{p}<0.05$ will be considered statistically significant. We will also report additional relevant data, which may affect the study outcome. This will include lifestyle modifications, litigation cases, changes in socioeconomic status, physical symptoms and psychological status.

The measure of treatment effect will be changes in neuropsychological assessment scores. We will calculate the effect size of each S-NAB mean Domain Standard score (Attention, Language, Memory, Spatial and Executive Function domains) as well as the Total Index Score within each intervention group. Cohen's d moderate $(>0.5)$ to large effect size $(>0.8)$ is considered to be clinically significant. Another treatment effect analysis will include reporting on the CogniPlus Attention task difficulty level achieved for each program (ALERT, FOCUS, VIG, SELECT and DIVID), the change of response time and measurement of errors.

Similarly, functional changes will be measured by using the effect size calculation of mean GAS T scores obtained at preintervention and postintervention. We will also compare the mean change in GAS T score between groups and report on the type and preference of metacognitive strategies used by participants of both groups.

The secondary analysis will include measurement of structural brain changes following intervention. These data will be obtained from the DTI MRI scan performed at preintervention and postintervention, for all groups. We will identify FA, MD and RD parameters with statistically significant mean values $(p<0.05)$ via whole brain analysis known as Tract-Based Spatial Statistics (TBSS) ${ }^{80}$ and region of interest approach which is part of the FSL (V.5.0.6; University of Oxford, Oxford UK) ${ }^{81}$ and AFNI (V.2011_12_21_1014; National Institute of Mental Health, Bethesda, Maryland, USA) software packages. The DTI parameters of both intervention groups at 3 and 6 months study timelines will be compared with the healthy control group by using repeated measure analysis. This is in the assumption that the study fulfils the repeated measure analysis of normally distributed data sample and homogeneity of variance.

Further analysis also includes correlation of cognitive performance with structural brain changes. We will perform Pearson's correlation coefficient between mean S-NAB Standard score of each domain and the selected WMT (with statistical significant).

\section{Data management}

All data obtained including from non-adherence or voluntarily withdrawn participants will also be reviewed and included in the study analysis where applicable. All study documents will be securely kept at the study site. Participant information will be stored in locked filing cabinets and will only be accessible to selected investigators. All data documents, administrative forms, reports and analysis documents will only have coded participant ID to avoid identification by any investigator of the study. Data entry will only be performed by an appointed research assistant. Any other document that has a participant's name such as consent form will be kept in a separate cabinet accessible by a selected investigator (MM).

\section{DISCUSSION}

To our knowledge, this is the first randomised control trial of cognitive intervention in adult mTBI population, conducted in a low/middle-income country, Southeast Asia region. Previous studies have been conducted in the Western population with a predominantly Caucasian ethic group and limited ethnic variation. A study from this region with various ethnic group involvements of both genders may better represent the study population and in turn add further knowledge on the pattern of impairment following mTBI. Uniquely, cultural practice and belief system may also influence treatment response and outcome. Development of the intervention approach was based on current evidence, a pilot study and expert panel review. This trial incorporates technology in the treatment application, consistent with the changing face of health service delivery in Malaysia, aiming at resource efficiency and treatment effectiveness, although using a tailored treatment approach appropriate for the local setting. The results of this study will provide a comprehensive overview on the effect of cognitive rehabilitation in mTBI. Owing to the paucity of scientific and clinical knowledge, this trial will also contribute to the evidencebased cognitive treatment model for mTBI population.

\section{Trial status}

At the time of manuscript preparation, 30 potential participants have been recruited at 3 months post-injury. Fifteen participants were consented and received treatment following randomisation. Recruitment is due to finish in April 2019. Data lock has not yet occurred and no analyses have been performed.

\section{Author affiliations}

${ }^{1}$ Department of Rehabilitation Medicine, Faculty of Medicine, University of Malaya, Kuala Lumpur, Malaysia

${ }^{2}$ Department of Surgery, Faculty of Medicine, University of Malaya, Kuala Lumpur, Malaysia

${ }^{3}$ Department of Biomedical Imaging, Faculty of Medicine, University of Malaya, Kuala Lumpur, Malaysia

${ }^{4}$ Department of Rehabilitation Medicine, University of Malaya Medical Centre, Kuala Lumpur, Malaysia

${ }^{5}$ Department of Social and Preventive Medicine, Faculty of Medicine, University of Malaya, Kuala Lumpur, Malaysia 
${ }^{6}$ Institute for Public Health, National Institutes of Health, Ministry of Health, Setia Alam, Malaysia

${ }^{7}$ Faculty of Medicine and Health Sciences, University of Nottingham, Nottingham, United Kingdom

${ }^{8}$ School of Medicine, University of Nottingham, Nottingham, United Kingdom ${ }^{9}$ Institute of Mental Health, Nottinghamshire Healthcare Trust, Nottingham, United Kingdom

${ }^{10}$ Lee Kong Chian Faculty of Engineering and Science, Universiti Tunku Abdul Rahman, Sungai Long Campus, Malaysia

Acknowledgements We wish to thank all our mTBI participants involved in the pilot control study as well as expert panels in involved in the review of our intervention development and study.

Contributors NH initiated the study, applied for study funding and is the principal investigator. NH, MM, VN, NR, AD, RdN and SYG were involved in the conception, development of the intervention and design of the study. NAM and NAMT implemented the cognitive intervention. LKT provided the consultation on DTI processing and analysis. MD and NAM provided important statistical contributions. All authors provided feedback on drafts of this paper, read and approved the final manuscript. NH, MM, VN and NR are the guarantors for the study and accept full responsibility for the work and/or the conduct of the study, had access to data, and controlled the decision to publish. MM is the corresponding author and attests that all listed authors meet authorship criteria and that no others meeting the criteria have been omitted.

Funding This study is partially funded by High Impact Research Grant UM.C/625/1/ HIR/MOHE/CHAN/12 and fully funded by Post Graduate Research Grant (IPPP) PPPC/ C1-2016/DGJ/01 and Malaysian Ministry of Science and Innovation (MOSTI) grant (MOSTI Flagship Project FP0911F001). Trial sponsor: University of Malaya, Malaysia.

Competing interests None declared.

Patient consent for publication Not required.

Ethics approval This study is approved by Medical Research Ethics Committee UMMC (MREC ID NO: 2016928-4293 and UM/EC Ref: 947.15) for the application of cognitive treatment on patients with $\mathrm{mTBI}$.

Provenance and peer review Not commissioned; externally peer reviewed.

Open access This is an open access article distributed in accordance with the Creative Commons Attribution Non Commercial (CC BY-NC 4.0) license, which permits others to distribute, remix, adapt, build upon this work non-commercially, and license their derivative works on different terms, provided the original work is properly cited, appropriate credit is given, any changes made indicated, and the use is non-commercial. See: http://creativecommons.org/licenses/by-nc/4.0/.

\section{REFERENCES}

1. Holm L, David Cassidy J, Carroll L, et al. Summary of the who collaborating centre for neurotrauma Task force on mild traumatic brain injury. J Rehabil Med 2005;37:137-41.

2. World Health Organization (WHO). Global status report on road safety 2015, 2015. Available: https://www.who.int/violence_injury_ prevention/road_safety_status/2015/en/ [Accessed Oct 2018].

3. Jamaluddin SF, Abd Wahab M, Abdul Wahab MY, et al. National trauma database January 2009 to December 2009- fourth report, 2011. Available: http://www.acrm.org.my/ntrd/report/ntrdReport 2009.pdf [Accessed July 2011].

4. Department of Statistics Malaysia. Statistics on causes of death, Malaysia, 2018, 2018. Available: https://www.dosm.gov.my/v1/index. php? $r=$ column/cthemeByCat\&cat=401\&bul_id=aWg2VjJkZHhYcDdE M3JQSGloeTVIZz09\&menu_id=LOpheU43NWJWRWVSZkIWdzQ4 TIhUUT09 [Accessed 31 Oct 2018].

5. Carroll L, Cassidy JD, Peloso P, et al. Prognosis for mild traumatic brain injury: results of the who collaborating centre Task force on mild traumatic brain injury. J Rehabil Med 2004;36:84-105.

6. Ponsford Jet al. Impact of early intervention on outcome following mild head injury in adults. J Neurol, Neurosurg Psychiatry 2002;73:330-2.

7. Belanger HG, Curtiss G, Demery JA, et al. Factors moderating neuropsychological outcomes following mild traumatic brain injury: a meta-analysis. J Int Neuropsychol Soc 2005;11:215-27.

8. Heitger MH, Jones RD, Dalrymple-Alford JC, et al. Motor deficits and recovery during the first year following mild closed head injury. Brain Inj 2006;20:807-24.
9. Landre N, Poppe C, Davis N, et al. Cognitive functioning and postconcussive symptoms in trauma patients with and without mild TBI. Arch Clin Neuropsychol 2006;21:255-73.

10. Stulemeijer M, van der Werf S, Borm GF, et al. Early prediction of favourable recovery 6 months after mild traumatic brain injury. $J$ Neurol Neurosurg Psychiatry 2008;79:936-42.

11. Rohling ML, Binder LM, Demakis GJ, et al. A meta-analysis of neuropsychological outcome after mild traumatic brain injury: reanalyses and reconsiderations of binder et al. (1997), Frencham et al. (2005), and Pertab et al. (2009). Clin Neuropsychol 2011;25:608-23.

12. Cassidy JD, Cancelliere C, Carroll LJ, et al. Systematic review of self-reported prognosis in adults after mild traumatic brain injury: results of the International collaboration on mild traumatic brain injury prognosis. Arch Phys Med Rehabil 2014;95:S132-51.

13. Königs M, de Kieviet JF, Oosterlaan J. Post-Traumatic amnesia predicts intelligence impairment following traumatic brain injury: a meta-analysis. J Neurol Neurosurg Psychiatry 2012;83:1048-55.

14. Brewer TL, Metzger BL, Therrien B. Trajectories of cognitive recovery following a minor brain injury. Res Nurs Health 2002;25:269-81.

15. Bennett TL, Dittmar C, Ho M. The neuropsychology of traumatic brain injury. In: Horton Jr AM, Wedding D, Webster J, eds. The neuropsychology Handbook: behavioral and clinical perspectives. New York: Springer Publishing Company, 1997: 123-72.

16. Tsaousides T, Gordon WA. Cognitive rehabilitation following traumatic brain injury: assessment to treatment. Mt Sinai J Med 2009;76:173-81.

17. Haskins EC, Cicerone K, Dams-O'Connor K, et al. Cognitive rehabilitation manual translating evidence-based recommendations into practice. Virginia, USA: ACRM Publishing, 2014.

18. Warden DL, Gordon B, Neurobehavioural Guidelines Working Group. Neurobehavioral guidelines Working Group. guidelines for the pharmacologic treatment of neurobehavioral sequelae of traumatic brain injury. J Neurotrauma 2006;23:1468-501.

19. Prince $C$, Bruhns M. Evaluation and treatment of mild traumatic brain injury: the role of neuropsychology. Brain Sci 2017;7:105-14.

20. Donovan J, Cancelliere C, Cassidy JD. Summary of the findings of the International collaboration on mild traumatic brain injury prognosis. Chiropr Man Therap 2014;22.

21. Karr JE, Areshenkoff CN, Garcia-Barrera MA. The neuropsychological outcomes of concussion: a systematic review of meta-analyses on the cognitive sequelae of mild traumatic brain injury. Neuropsychology 2014;28:321-36.

22. Pertab JL, James KM, Bigler ED. Limitations of mild traumatic brain injury meta-analyses. Brain Injury 2009;23:498-508.

23. Hartvigsen J, Boyle E, Cassidy JD, et al. Mild traumatic brain injury after motor vehicle collisions: what are the symptoms and who treats them? a population-based 1-year inception cohort study. Arch Phys Med Rehabil 2014;95:S286-S294.

24. Veeramuthu V, Narayanan V, Kuo TL, et al. Diffusion tensor imaging parameters in mild traumatic brain injury and its correlation with early neuropsychological impairment: a longitudinal study. J Neurotrauma 2015;32:1497-509.

25. Theadom A, Parag V, Dowell T, et al. Persistent problems 1 year after mild traumatic brain injury: a longitudinal population study in New Zealand. Br J Gen Pract 2016;66:e16-23.

26. De Silva MJ, Roberts I, Perel P, et al. Patient outcome after traumatic brain injury in high-, middle- and low-income countries: analysis of data on 8927 patients in 46 countries. Int J Epidemiol 2009;38:452-8.

27. New South Wales Motor Accident Authority. Guidelines for mild traumatic brain injury following closed head injury, 2008. Available: http://braininjuryhelp.ca/wp-content/uploads/2014/04/NSW-MAAGuidelines_for_Mild_Traumatic_Brain_Injury_Following_Closed Head_Injury_1.pdf [Äccessed Jul 2017].

28. Marshall S, Bayley M, McCullagh S, et al. Updated clinical practice guidelines for concussion/mild traumatic brain injury and persistent symptoms. Brain Injury 2015;29:688-700.

29. Kashluba S, Hanks RA, Casey JE, et al. Neuropsychologic and functional outcome after complicated mild traumatic brain injury. Arch Phys Med Rehabil 2008;89:904-11.

30. Cicerone KD, Langenbahn DM, Braden C, et al. Evidence-Based cognitive rehabilitation: updated review of the literature from 2003 through 2008. Arch Phys Med Rehabil 2011;92:519-30.

31. Lu J, Gary KW, Neimeier JP, et al. Randomized controlled trials in adult traumatic brain injury. Brain Inj 2012;26:1523-48.

32. Bajo A, Fleminger S. Brain injury rehabilitation: what works for whom and when? Brain Inj 2002;16:385-95.

33. Chan RCK. Attentional deficits in patients with post-concussion symptoms: a componential perspective. Brain Inj 2001;15:71-94.

34. Cappa SF, Benke T, Clarke S, et al. EFNS guidelines on cognitive rehabilitation: report of an EFNS Task force. Eur $J$ Neurol 2005;12:665-80. 
35. Comper P, Bisschop SM, Carnide N, et al. A systematic review of treatments for mild traumatic brain injury. Brain Injury 2005;19:863-80.

36. Snell DL, Surgenor LJ, Hay-Smith EJC, et al. A systematic review of psychological treatments for mild traumatic brain injury: an update on the evidence. J Clin Exp Neuropsychol 2009;31:20-38.

37. van Heugten C, Wolters Gregório G, Wade D. EvidenceBased cognitive rehabilitation after acquired brain injury: a systematic review of content of treatment. Neuropsychol Rehabil 2012;22:653-73.

38. Zickefoose S, Hux K, Brown J, et al. Let the games begin: A preliminary study using Attention Process Training-3 and Lumosity TM brain games to remediate attention deficits following traumatic brain injury. Brain Injury 2013;27:707-16.

39. Johansson B, Tornmalm M. Working memory training for patients with acquired brain injury: effects in daily life. Scand J Occup Ther 2012;19:176-83.

40. Lebowitz MS, Dams-O Connor K, Cantor JB. Feasibility of computerized brain plasticity-based cognitive training after traumatic brain injury. JRRD 2012;49:1547-56.

41. Serino A, Ciaramelli E, Santantonio AD, et al. A pilot study for rehabilitation of central executive deficits after traumatic brain injury. Brain Injury 2007;21:11-19.

42. Youse KM, Coelho CA. Treating underlying attention deficits as a means for improving conversational discourse in individuals with closed head injury: a preliminary study. Neurorehabil 2009;24:355-64.

43. Dirette D. A comparison of attention, processing and strategy use by adults with and without acquired brain injuries. Brain Injury 2004;18:1219-27.

44. Cicerone KD, Goldin Y, Ganci K, et al. Evidence-Based cognitive rehabilitation: systematic review of the literature from 2009 through 2014. Arch Phys Med Rehabil 2019;100:1515-33.

45. Kallweit D, CogniPlus EJ. Training cognitive functions. Moedling, Austria: Paul Gerin Druckerei, 2004

46. Bogdanova Y, Yee MK, Ho VT, et al. Computerized cognitive rehabilitation of attention and executive function in acquired brain injury: a systematic review. J Head Trauma Rehabil 2016;31:419-33

47. Cerasa A, Gioia MC, Valentino P, et al. Computer-Assisted cognitive rehabilitation of attention deficits for multiple sclerosis: a randomized trial with fMRI correlates. Neurorehabil Neural Repair 2013;27:284-95.

48. Hwi-young C, Ki-Tae K, Jin-Hwa J. Effects of computer assisted cognitive rehabilitation on brain wave, memory and attention of stroke patients: a randomized control trial. J Phys Ther Sci 2015;27:1029-32.

49. Sohlberg MM, Mateer CA. Improving attention and managing attentional problems. adapting rehabilitation techniques to adults with add. Ann N Y Acad Sci 2001;931:359-75.

50. Waid-Ebbs JK, Daly J, Wu SS, et al. Response to goal management training in veterans with blast-related mild traumatic brain injury. $J$ Rehabil Res Dev 2014:51:1555-66.

51. Twamley EW, Jak AJ, Delis DC, et al. Cognitive symptom management and rehabilitation therapy (CogSMART) for veterans with traumatic brain injury: pilot randomized controlled trial. J Rehabil Res Dev 2014;51:59-70.

52. Huckans M, Pavawalla S, Demadura T, et al. A pilot study examining effects of group-based cognitive strategy training treatment on self-reported cognitive problems, psychiatric symptoms, functioning, and compensatory strategy use in OIF/OEF combat veterans with persistent mild cognitive disorder and history of traumatic brain injury. JRRD 2010;47:43-60.

53. Rasquin SMC, Bouwens SFM, Dijcks B, et al. Effectiveness of a low intensity outpatient cognitive rehabilitation programme for patients in the chronic phase after acquired brain injury. Neuropsychol Rehabil 2010;20:760-77.

54. McKerracher G, Powell T, Oyebode J. A single case experimental design comparing two memory notebook formats for a man with memory problems caused by traumatic brain injury. Neuropsychol Rehabil 2005;15:115-28.

55. Laatsch L, Stress M. Neuropsychological change following individualized cognitive rehabilitation therapy. NeuroRehabil 2000;15:189-97.

56. Walker JP. Functional outcome: a case for mild traumatic brain injury Brain Injury 2002;16:611-25.
57. Cicerone KD. Remediation of 'working attention' in mild traumatic brain injury. Brain Injury 2002;16:185-95.

58. Niemeier JP, Kreutzer JS, Taylor LA. Acute cognitive and neurobehavioural intervention for individuals with acquired brain injury: preliminary outcome data. Neuropsychol Rehabil 2005;15:129-46.

59. Park NW, Ingles JL. Effectiveness of attention rehabilitation after an acquired brain injury: a meta-analysis. Neuropsychology 2001;15:199-210.

60. Bigler ED. Neuroimaging biomarkers in mild traumatic brain injury (mTBI). Neuropsychol Rev 2013;23:169-209.

61. Stern RA, White T. Neuropsychological Assessment Battery (NAB) Administration, Scoring and Interpretation Manual. 2nd edn. Lutz, FL: PAR, 2001

62. Zgaljardic DJ, Temple RO. Reliability and validity of the neuropsychological assessment Battery-Screening module (NAB$\mathrm{SM}$ ) in a sample of patients with moderate-to-severe acquired brain injury. Appl Neuropsychol 2010;17:27-36.

63. Temple RO, Zgaljardic DJ, Abreu BC, et al. Ecological validity of the neuropsychological assessment battery screening module in postacute brain injury rehabilitation. Brain Injury 2009;23:45-50.

64. Donders J, Levitt T. Criterion validity of the neuropsychological assessment battery after traumatic brain injury. Archives of Clinical Neuropsychology 2012;27:440-5

65. Shenton ME, Hamoda HM, Schneiderman JS, et al. A review of magnetic resonance imaging and diffusion tensor imaging findings in mild traumatic brain injury. Brain Imaging Behav 2012;6:137-92.

66. Hulkower MB, Poliak DB, Rosenbaum SB, et al. A decade of DTI in traumatic brain injury: 10 years and 100 articles later. AJNR Am J Neuroradiol 2013;34:2064-74

67. Niogi SN, Mukherjee P, Ghajar J, et al. Extent of microstructural white matter injury in Postconcussive syndrome correlates with impaired cognitive reaction time: a 3T diffusion tensor imaging study of mild traumatic brain injury. American Journal of Neuroradiology 2008;29:967-73

68. Yurgelun-Todd DA, Bueler CE, McGlade EC, et al. Neuroimaging correlates of traumatic brain injury and suicidal behavior. Journal of Head Trauma Rehabilitation 2011;26:276-89.

69. Holli KK, Waljas M, Harrison L, et al. Mild traumatic brain injury: tissue texture analysis correlated to neuropsychological and DTI findings. Academic Radiology 2010;17:1096-102.

70. Lipton ML, Gulko E, Zimmerman ME, et al. Diffusion-tensor imaging implicates prefrontal axonal injury in executive function impairment following very mild traumatic brain injury. Radiology 2009;252:816-24.

71. Rockwood K, Joyce B, Stolee P. Use of goal attainment scaling in measuring clinically important change in cognitive rehabilitation patients. J Clin Epidemiol 1997;50:581-8.

72. Grant M, Ponsford J. Goal attainment scaling in brain injury rehabilitation: strengths, limitations and recommendations for future applications. Neuropsychol Rehabil 2014;24:661-77.

73. Sander A. The extended Glasgow outcome scale. The center for outcome measurement in brain injury, 2002. Available: http://www. tbims.org/combi/gose [Accessed 9 Apr 2018].

74. Wright $J$. The functional assessment measure. The center for outcome measurement in brain injury, 2000. Available: http://www. tbims.org/FIM [Accessed 9 Apr 2018]

75. Hart T. The moss attention rating scale. The center for outcome measurement in brain injury. Available: http://tbims.org/mars/index. html [Accessed 9 Apr 2018]

76. Hurn J, Kneebone I, Cropley M. Goal setting as an outcome measure: a systematic review. Clin Rehabil 2006;20:756-72.

77. Kiresuk TJ, Sherman RE. Goal attainment scaling: a general method for evaluating comprehensive community mental health programs. Community Ment Health J 1968;4:443-53.

78. Kiresuk T, Smith A, Cardillo J. Goal attainment scaling: application, theory and measurement. New York: Lawrence Erlbaum Associates, 1994.

79. Boman $\mathrm{I}-\mathrm{L}$, Lindstedt $\mathrm{M}$, Hemmingsson $\mathrm{H}$, et al. Cognitive training in home environment. Brain Inj 2004;18:985-95.

80. Smith SM, Jenkinson M, Johansen-Berg H, et al. Tract-based spatial statistics: voxelwise analysis of multi-subject diffusion data. Neuroimage 2006;31:1487-505.

81. Smith SM, Jenkinson M, Woolrich MW, et al. Advances in functional and structural $\mathrm{Mr}$ image analysis and implementation as fsl. Neuroimage 2004;23:S208-S219. 